\title{
Identification of Potential Long Non-Coding RNA Candidates that Contribute to Triple-Negative Breast Cancer in Humans through Computational Approach
}

\author{
Md. Motiar Rahman ${ }^{1,2, *,+}$ (D), Md. Tofazzal Hossain $3,4,5,+$, Md. Selim Reza ${ }^{3,4}$, Yin Peng ${ }^{6}$, Shengzhong Feng 4 \\ and Yanjie Wei ${ }^{4, *}$ \\ 1 Department of Biochemistry and Molecular Biology, University of Rajshahi, Rajshahi 6205, Bangladesh \\ 2 Department of Chemistry, Binghamton University, State University of New York, Vestal, New York, \\ NY 13902, USA \\ 3 University of Chinese Academy of Sciences, No.19(A) Yuquan Road, Shijingshan District, Beijing 100049, \\ China; tofazzal.stat@gmail.com (T.H.); selim@siat.ac.cn (S.R.) \\ 4 Joint Engineering Research Center for Health Big Data Intelligent Analysis Technology, Shenzhen Institutes of \\ Advanced Technology, Chinese Academy of Sciences, Shenzhen 518055, China; sz.feng@siat.ac.cn \\ 5 Department of Statistics, Bangabandhu Sheikh Mujibur Rahaman Science and Technology University, \\ Gopalganj 8100, Bangladesh \\ 6 Department of Pathology, The Shenzhen University School of Medicine, Shenzhen 518060, China; \\ ypeng@szu.edu.cn \\ * Correspondence: mrahman@binghamton.edu (M.R.); yj.wei@siat.ac.cn (Y.W.) \\ + These authors have equal contribution.
}

check for

updates

Citation: Rahman, M.M.; Hossain, M.T.; Reza, M.S.; Peng, Y.; Feng, S.; Wei, Y. Identification of Potential Long Non-Coding RNA Candidates that Contribute to Triple-Negative Breast Cancer in Humans through Computational Approach. Int. J. Mol. Sci. 2021, 22, 12359. https://doi.org/ $10.3390 /$ ijms222212359

Academic Editor: Monica Cantile

Received: 6 October 2021

Accepted: 28 October 2021

Published: 16 November 2021

Publisher's Note: MDPI stays neutral with regard to jurisdictional claims in published maps and institutional affiliations.

Copyright: (c) 2021 by the authors. Licensee MDPI, Basel, Switzerland. This article is an open access article distributed under the terms and conditions of the Creative Commons Attribution (CC BY) license (https:/ / creativecommons.org/licenses/by/ $4.0 /)$.

\begin{abstract}
Breast cancer (BC) is the most frequent malignancy identified in adult females, resulting in enormous financial losses worldwide. Owing to the heterogeneity as well as various molecular subtypes, the molecular pathways underlying carcinogenesis in various forms of BC are distinct. Therefore, the advancement of alternative therapy is required to combat the ailment. Recent analyses propose that long non-coding RNAs (lncRNAs) perform an essential function in controlling immune response, and therefore, may provide essential information about the disorder. However, their function in patients with triple-negative BC (TNBC) has not been explored in detail. Here, we analyzed the changes in the genomic expression of messenger RNA (mRNA) and lncRNA in standard control in response to cancer metastasis using publicly available single-cell RNA-Seq data. We identified a total of 197 potentially novel lncRNAs in TNBC patients of which 86 were differentially upregulated and 111 were differentially downregulated. In addition, among the 909 candidate lncRNA transcripts, 19 were significantly differentially expressed (DE) of which three were upregulated and 16 were downregulated. On the other hand, 1901 mRNA transcripts were significantly DE of which 1110 were upregulated and 791 were downregulated by TNBCs subtypes. The Gene Ontology (GO) analyses showed that some of the host genes were enriched in various biological, molecular, and cellular functions. The Kyoto encyclopedia of genes and genomes (KEGG) pathway analysis showed that some of the genes were involved in only one pathway of prostate cancer. The lncRNA-miRNA-gene network analysis showed that the lncRNAs TCONS_00076394 and TCONS_00051377 interacted with breast cancer-related micro RNAs (miRNAs) and the host genes of these lncRNAs were also functionally related to breast cancer. Thus, this study provides novel lncRNAs as potential biomarkers for the therapeutic intervention of this cancer subtype.
\end{abstract}

Keywords: long non-coding RNA; lncRNA biomarker; triple-negative breast cancer

\section{Introduction}

$\mathrm{BC}$ is regarded as the most frequently observed cancer in females throughout the world [1-3], and distance metastasis is the foremost cause of lower survival rate [4,5]. TNBC is a subgroup of BCs that lack the expression of estrogen receptor (ER), and progesterone 
receptor (PR), as well as human epidermal growth factor receptor 2 (HER-2) [6,7]. In addition, the possibility of distant metastasis along with the recurrence in patients with TNBC is relatively higher when compared to normal patients [8]. So the management strategies of TNBC are somewhat difficult due to the comparatively aggressive biological features and deficiency of distinct targeted medication [9]. Therefore, there is a need to acquire a good understanding of the mechanisms and regulations of tumorigenesis in TNBC cells as well as the elucidation of efficient biomarkers for the prognosis and diagnosis of TNBC patients. To this line, identification of the novel metastasis-related molecular pathway is of great importance to developing the consequence of TNBC treatments.

Recently, it was revealed that long non-coding RNAs (lncRNAs) have close interactions with BC metastasis [10-13]. LncRNAs are a class of non-coding RNAs with a length of $>200$ nucleotides, which are linked with many biological processes, such as cancer cell invasion, proliferation, apoptosis, differentiation, development, and metastasis $[14,15]$. So far, IncRNAs have been exhibited to play auxiliary roles either to tumorigenesis or to tumor suppression. Specifically, in BC, increasing data has strengthened the hypothesis that lncRNAs exert an essential function in controlling BC metastasis [16-18]. Many lncRNAs were underregulated abnormally in a variety of BC cells [3,19-21], ovarian cancer [22], hepatocellular carcinoma [23], and many others [14,24]. Numerous studies have suggested that various lncRNAs, including HOTAIR, BCAR4, and linc-ROR, were upregulated and stimulated BC invasion as well as metastasis. HOTAIR upregulation was shown to be strongly related to lymph node metastasis in patients with TNBCs [25], despite the fact of how it controls lymph node metastasis, as well as BC lung metastasis, are not well understood. Therefore, it demands extensive studies about the roles of lncRNA in TNBCs.

Latest studies have discovered that lncRNAs play a crucial role in the tumorigenesis and progression in TNBC. SOX21-AS1, HOST2, HUMT, XIST, FAM83H-AS1, and LINC00173, for example, were reported as potential candidates in the initiation and development of TNBC [26-30]. Several other studies have identified AFAP1-AS1, MALAT1, NRON, and RMST as significant regulators of TNBC-mediated cell proliferation, migration, metastasis, and tumorigenicity [31-34]. Similarly, lncRNA MIR100HG and LINC01638 regulate cell proliferation [35,36], MTDH enhances breast tumorigenicity [37] and SNHG12 regulates cell proliferation and migration in such breast cancer types [38].

It was exhibited that lncRNAs may function as agonists or antagonists of transcription, protein scaffolds, miRNA sponges, or antisense RNA [39]. They are recognized to have lower evolutionary conservation and show lower cellular concentration compared to protein-coding transcripts, but possess a higher degree of tissue specificity [40]. Nevertheless, despite having an outstanding role in the regulation of gene expression, lncRNAs were poorly identified and annotated in TNBC patients [41]. The objective of this study is to deepen the knowledge about the crucial interplay between lncRNAs expression and TNBCs and provide novel insight into the regulatory function of lncRNAs in humans during TNBCs. To this end, we used the bioinformatics pipeline to identify the lncRNAs expression profiles and their putative role in TNBCs patients using publicly available single-cell RNA-seq data.

\section{Results}

\subsection{Identification of $\operatorname{lnc} R N A$ Transcripts}

We analyzed scRNASeq data of 60 TNBC samples collected from six patients (10 samples per patient) and 20 normal samples to identify potential lncRNAs responsible for TNBCs. A total of 83,401 transcripts from 80 samples were identified. Firstly, 10,385 transcripts with a class code " $j$ " having unknown annotations were set for selecting the putative IncRNAs. Then, a filtering strategy was adopted to filter out low-quality assemblies such as those having $<200 \mathrm{bp}$ for more than one exon transcript and $<500 \mathrm{bp}$ for single-exon transcripts. The minimum length of the transcripts with a class " $j$ " was 255 and there were no single-exon transcripts. Therefore, all the transcripts with a class code " $j$ " passed the filtering step. The protein-coding ability of the 10,385 transcripts was assessed by the 
coding potential calculator 2 (CPC2). The threshold cut-off for coding probability $p<0.5$ was used to find the non-coding candidate transcripts as the standard rule of CPC2 is to declare any transcript having a coding probability of $p<0.5$ as "non-coding" and "coding" otherwise. Finally, a total of 909 candidate lncRNAs were detected.

\subsection{Expression Profiles of $\ln \mathrm{R} N A$ As and $m R N A s$}

In comparison to other RNAs, IncRNAs have been demonstrated to exhibit distinct characteristics, such as lower levels of cellular concentration, fewer exons, and shorter length than protein-coding genes [41]. In this study, we compared the number of exons and their length, chromosome distribution, and expression between lncRNAs and mRNAs in our dataset. It was shown that the number of mRNA exons (median 9) was greater than that of lncRNAs exons (median 5), and the number was statistically significant which was determined by a two-tailed Mann-Whitney U-test $\left(p=2.20 \times 10^{-16}\right)$. We also observed, in lncRNAs, about $66 \%$ of the transcripts having $\leq 6$ exons and $34 \%$ of the transcripts having $\leq 4$ exons. The length of IncRNAs transcript (median 1899) was smaller than that of mRNAs (median 2780) with $p=2.20 \times 10^{-16}$ determined by a two-tailed Mann-Whitney U-test. Similarly, the level of lncRNAs expression was smaller than that of mRNAs with mean FPKM values 6.53 and 17.13, respectively. The Student's $t$-test provided evidence that the difference in expression levels between lncRNAs (mean FPKM 6.53) and mRNA (mean FPKM 17.13) was statistically significant with $p=0.01625$. The distribution of lncRNAs across chromosomes was heterogeneous, and most of the lncRNAs exist in chr1 (77/909, $8.47 \%$ ). Compared to other chromosomes, the chr1, chr2, chr3, chr7, chr10, chr11, and chr19 possessed more lncRNAs, which accounted for more than $5 \%$ of total potential lncRNAs in each chromosome and the average number of lncRNAs per chromosome was 34. The expression profiles of lncRNAs and mRNAs have been shown in Figure 1.

\subsection{Differential Expression Analysis of IncRNAs and $m R N A s$}

We next identified the differentially expressed (DE) lncRNAs and mRNAs between two groups (normal vs. cancer). The R package DESeq was applied for this purpose. The volcano plot was used to find out the DE lncRNAs and mRNAs with the thresholds $p$-value $<0.05$ and foldchange $>2.0$ (Figure 2). Out of a total of 55,728 mRNA transcripts, 1901 mRNA transcripts were significantly DE. Among the significant DE mRNA transcripts, 1110 were upregulated and 791 were downregulated. On the other hand, out of a total of 15,308 lncRNA transcripts, 197 transcripts were significantly DE of which 86 were upregulated and 111 were downregulated. Similarly, among the 909 candidate lncRNA transcripts, 19 were significantly DE of which three were upregulated and 16 were downregulated. Tables 1 and 2 represented the top 20 DE (10 upregulated and 10 downregulated) transcripts for lncRNA and mRNA, respectively. In addition, the top 10 DE transcripts (three upregulated and seven downregulated) for candidate lncRNAs were given in Table 3. The expression patterns of lncRNA and mRNA transcripts were shown in the heatmap (Figure 3). The dendrograms of the two groups (tumor vs. normal) were clearly distinguishable. For the heatmap, 10 normal and 10 cancer samples were generated by averaging 20 and 60 samples, respectively. 

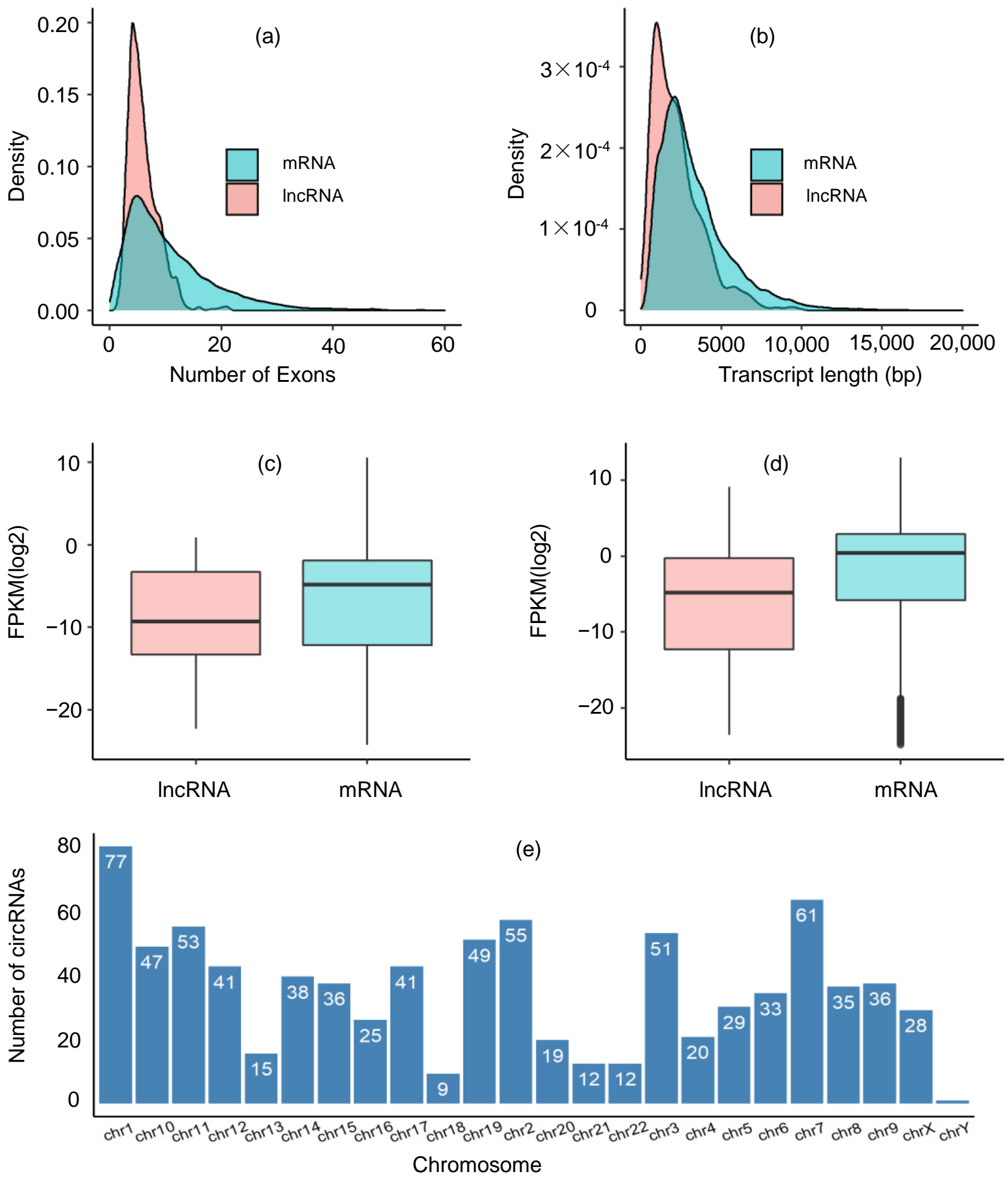

Figure 1. Expression profile of candidate lncRNA transcripts. Density plot for the number of exons in mRNA and lncRNA transcripts (a), the length of mRNA and lncRNA transcripts (b), expression levels (FPKM) of mRNA and lncRNA transcripts in normal samples (c), expression levels (FPKM) of mRNA and lncRNA transcripts in TNBC patients (d) and chromosome distribution of the candidate lncRNAs (e). 

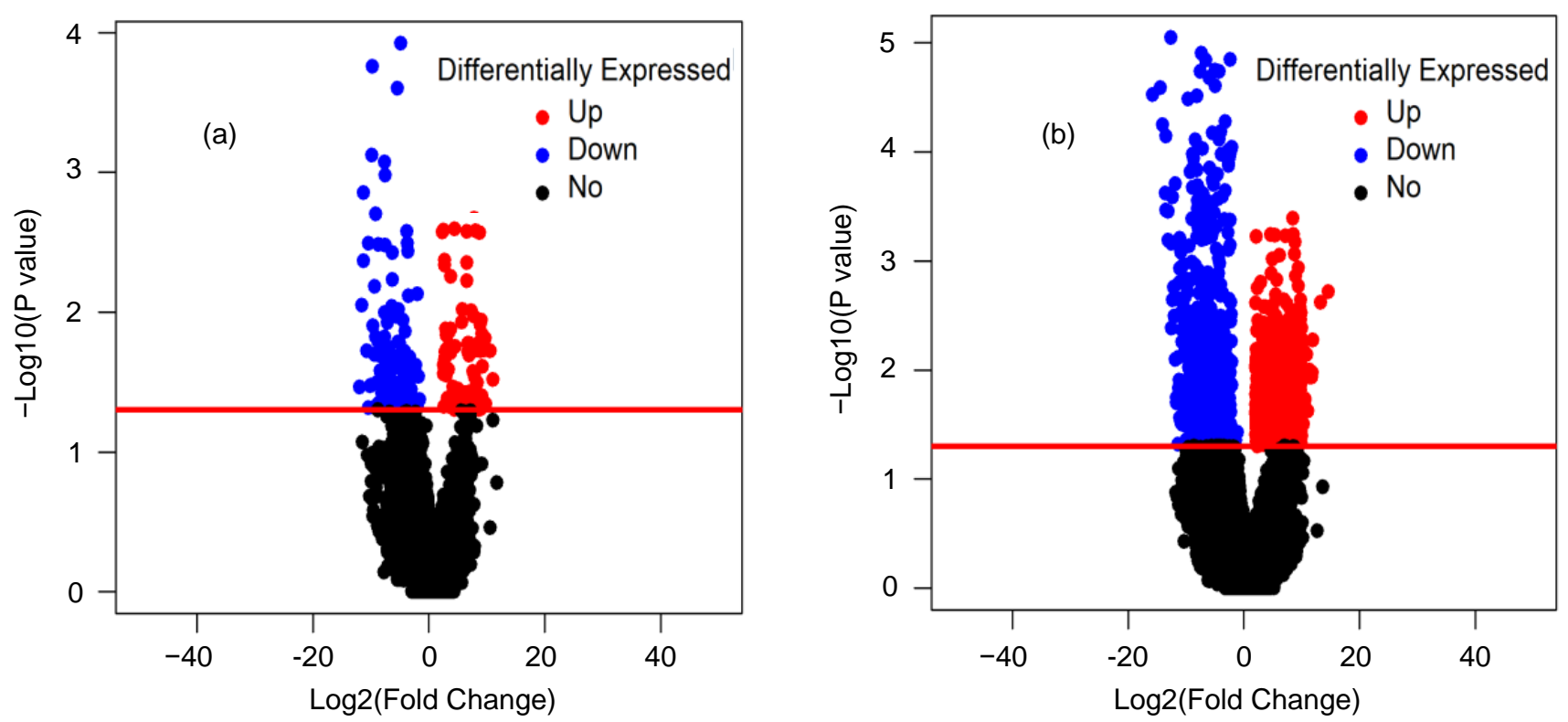

Figure 2. Volcano plot for the output of DESeq. LncRNA transcripts (a) and mRNA transcripts (b).

Table 1. Top 20 (10 upregulated and 10 downregulated) DE mRNAs. DE mRNAs were sorted based on $p$-value and fold change.

\begin{tabular}{lccccccc}
\hline mRNA ID & Location & No. of Exons & Length & Base Mean & LFC & $p$-Value & Gene \\
\hline TCONS_00027847 & chr16:72054592-72061056 & 7 & 1448 & 3419.94 & Inf & 0.00041 & HP \\
TCONS_00062848 & chr5:150401637-150412936 & 9 & 1681 & 2201.54 & Inf & 0.00058 & CD74 \\
TCONS_00027845 & chr16:72054592-72061056 & 5 & 1271 & 2210.50 & Inf & 0.00058 & HP \\
TCONS_00062849 & chr5:150401637-150412936 & 8 & 1489 & 2166.03 & Inf & 0.00058 & CD74 \\
TCONS_00037794 & chr19:6677835-6720682 & 41 & 5132 & 2131.98 & Inf & 0.00059 & C3 \\
TCONS_00027846 & chr16:72054592-72061056 & 5 & 1271 & 2125.11 & Inf & 0.00060 & HP \\
TCONS_00030302 & chr17:34255277-34257201 & 3 & 747 & 1907.13 & Inf & 0.00067 & CCL2 \\
TCONS_00008098 & chr10:58513144-58831437 & 21 & 5471 & 1317.61 & Inf & 0.00087 & BICC1 \\
TCONS_00062847 & chr5:150401637-150412936 & 6 & 1305 & 1274.15 & Inf & 0.00089 & CD74 \\
TCONS_00005374 & chr1:89052304-89065360 & 11 & 3050 & 1164.10 & Inf & 0.00096 & GBP1 \\
TCONS_00065563 & chr6:27866792-27867581 & 1 & 790 & 301.37 & -12.6 & $8.98 \times 10^{-06}$ & HIST1H1B \\
TCONS_00028779 & chr16:30104810-30113557 & 10 & 1098 & 105.09 & - Inf & $1.25 \times 10^{-05}$ & GDPD3 \\
TCONS_00010969 & chr10:128096661-128126204 & 14 & 11,415 & 114.64 & - Inf & $1.44 \times 10^{-05}$ & MKI67 \\
TCONS_00010970 & chr10:128096661-128126204 & 15 & 12,495 & 119.33 & - Inf & $1.45 \times 10^{-05}$ & MKI67 \\
TCONS_00012090 & chr11:62270155-62273157 & 3 & 506 & 117.08 & - Inf & $1.80 \times 10^{-05}$ & SCGB2A2 \\
TCONS_00031424 & chr17:79778178-79787650 & 5 & 4265 & 42.19 & - Inf & $1.84 \times 10^{-05}$ & CBX2 \\
TCONS_00082965 & chrX:154651972-154653579 & 3 & 764 & 24.78 & - Inf & $1.85 \times 10^{-05}$ & CTAG2 \\
TCONS_00054384 & chr3:170037947-170085395 & 4 & 2013 & 18.08 & - Inf & $2.11 \times 10^{-05}$ & GPR160 \\
TCONS_00082964 & chrX:154651972-154653579 & 2 & 993 & 20.37 & - Inf & $2.50 \times 10^{-05}$ & CTAG2 \\
TCONS_00018204 & chr12:52806543-52814116 & 9 & 2147 & 45.76 & -14.4 & $2.61 \times 10^{-05}$ & KRT4 \\
\hline
\end{tabular}

LFC $=\log 2$ FoldChange and Inf $=$ Infinite.

Table 2. Top 20 (10 upregulated and 10 downregulated) DE lncRNAs. DE lncRNAs were sorted based on $p$-value.

\begin{tabular}{cccccccc}
\hline lncRNA ID & Location & No. of Exons & Length & Base Mean & LFC & $p$-Value & Gene \\
\hline TCONS_00006590 & chr1:169690665-169708856 & 7 & 2206 & 176.9738 & Inf & 0.00426 & SELL \\
TCONS_00057817 & chr4:78645994-78684501 & 4 & 4214 & 362.9557 & Inf & 0.00254 & LINC01094 \\
TCONS_00057819 & chr4:78645994-78684501 & 3 & 4120 & 352.7188 & Inf & 0.00260 & LINC01094 \\
TCONS_00057820 & chr4:78645994-78684501 & 3 & 4105 & 345.6054 & Inf & 0.00263 & LINC01094 \\
TCONS_00057818 & chr4:78645994-78684501 & 4 & 4192 & 339.0413 & Inf & 0.00266 & LINC01094 \\
TCONS_00057821 & chr4:78645994-78684501 & 3 & 4066 & 338.1632 & Inf & 0.00268 & LINC01094 \\
TCONS_00057816 & chr4:78645994-78684501 & 5 & 4286 & 331.5815 & Inf & 0.00270 & LINC01094 \\
\hline
\end{tabular}


Table 2. Cont.

\begin{tabular}{cccccccc}
\hline lncRNA ID & Location & No. of Exons & Length & Base Mean & LFC & $p$-Value & Gene \\
\hline TCONS_00062278 & chr5:91368632-91383373 & 8 & 4332 & 208.4584 & Inf & 0.00443 & ARRDC3 \\
TCONS_00062275 & chr5:91368632-91380297 & 8 & 4621 & 200.0444 & Inf & 0.00463 & ARRDC3 \\
TCONS_00009315 & chr10:17214239-17229985 & 3 & 1875 & 121.2627 & Inf & 0.00557 & VIM-AS1 \\
TCONS_00082872 & chrX:152708261-152714549 & 5 & 825 & 2.665319 & - Inf & 0.00012 & CSAG3 \\
TCONS_00081533 & chrX:152753921-152760222 & 5 & 825 & 2.704539 & - Inf & 0.00018 & CSAG3 \\
TCONS_00035347 & chr19:751113-764319 & 4 & 1048 & 12.04587 & - Inf & 0.00025 & MISP \\
TCONS_00017579 & chr12:6848808-6851930 & 5 & 1615 & 5.361052 & - Inf & 0.00076 & CDCA3 \\
TCONS_00017578 & chr12:6848808-6851930 & 6 & 1792 & 5.745075 & - Inf & 0.00085 & CDCA3 \\
TCONS_00012837 & chr11:111912736-111926871 & 5 & 1527 & 30.57432 & - Inf & 0.00105 & HSPB2-C11orf52 \\
TCONS_00024012 & chr15:43593834-43599406 & 9 & 2842 & 10.85619 & -11.3 & 0.00140 & CKMT1B \\
TCONS_00081534 & chrX:152753921-152760222 & 5 & 789 & 0.786254 & - Inf & 0.00198 & CSAG3 \\
TCONS_00082873 & chrX:152708261-152714549 & 5 & 789 & 0.968629 & - Inf & 0.00266 & CSAG3 \\
TCONS_00082981 & chrX:155061625-155071272 & 6 & 2755 & 2.456605 & - Inf & 0.00322 & MTCP1 \\
\hline
\end{tabular}

LFC $=\log 2$ FoldChange and Inf $=$ Infinite.

Table 3. Top 10 (3 upregulated and 7 downregulated) DE candidate lncRNAs.

\begin{tabular}{cccccccc}
\hline lncRNA ID & Location & No. of Exons & Length & Base Mean & LFC & $p$-Value & Gene \\
\hline TCONS_00076394 & chr8:102648777-102655902 & 4 & 2309 & 36.34 & Inf & 0.0146 & KLF10 \\
TCONS_00002947 & chr1:182789449-182830384 & 11 & 2728 & 32.38 & Inf & 0.0391 & NPL \\
TCONS_00051377 & chr22:17734140-17774665 & 5 & 1901 & 9.47 & Inf & 0.0434 & BID \\
TCONS_00082872 & chrX:152708261-152714549 & 5 & 825 & 2.67 & - Inf & 0.0001 & CSAG3 \\
TCONS_00081533 & chrX:152753921-152760222 & 5 & 825 & 2.70 & - Inf & 0.0002 & CSAG3 \\
TCONS_00082981 & chrX:155061625-155071272 & 6 & 2755 & 2.46 & - Inf & 0.0032 & MTCP1 \\
TCONS_00026822 & chr16:4788397-4796491 & 6 & 883 & 22.91 & -7.6 & 0.0033 & SMIM22 \\
TCONS_00049160 & chr20:47298126-47356889 & 5 & 1346 & 2.07 & -3.5 & 0.0076 & ZMYND8 \\
TCONS_00054549 & chr3:184361714-184368595 & 5 & 1723 & 4.02 & -6.8 & 0.0220 & POLR2H \\
TCONS_00054548 & chr3:184361714-184368595 & 6 & 1871 & 4.60 & -5.9 & 0.0233 & POLR2H \\
\hline
\end{tabular}

LFC $=\log 2$ FoldChange and Inf $=$ Infinite.

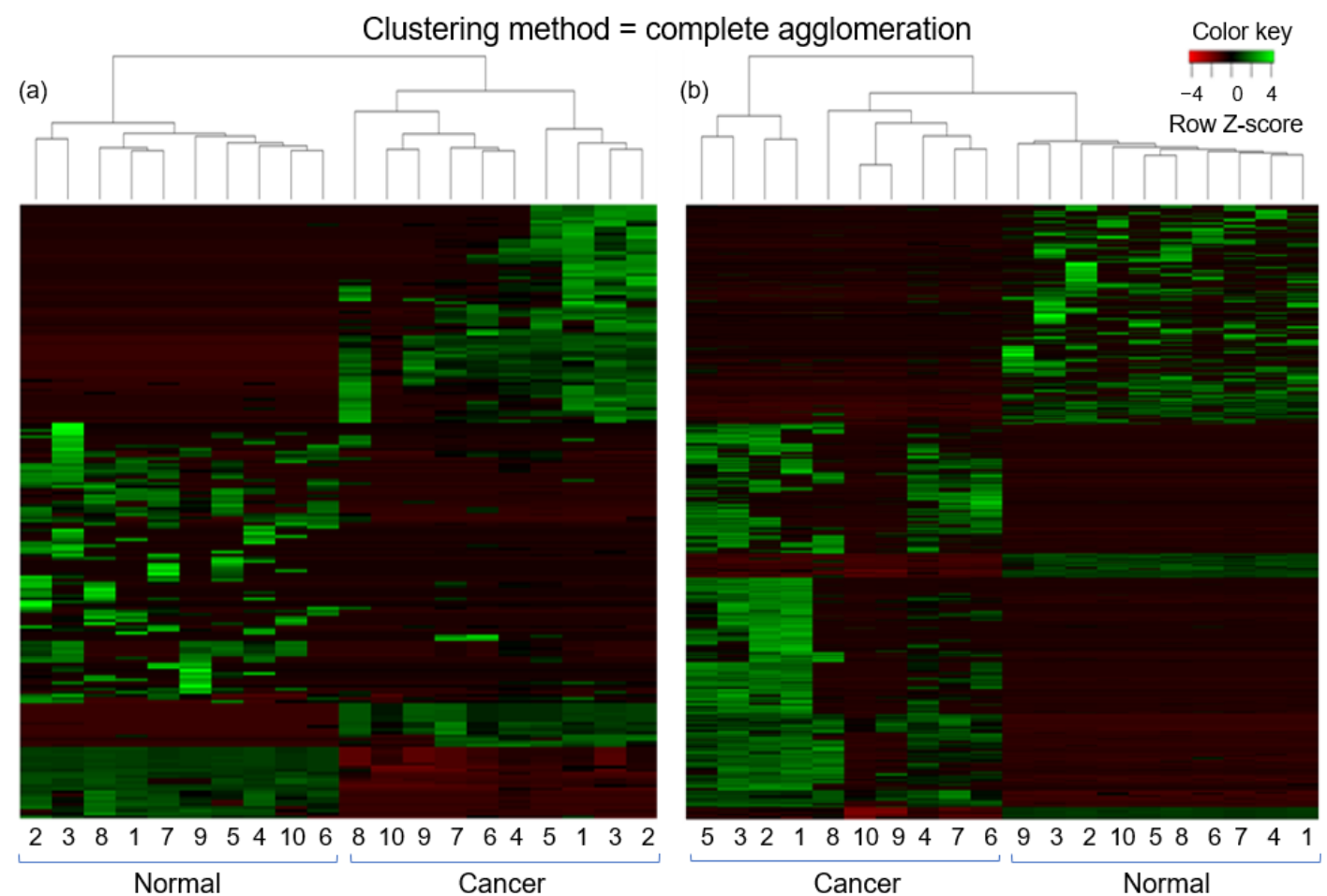

Figure 3. Heatmap of the DE transcripts. (a) lncRNA (b) mRNA. 


\subsection{Analysis of $\operatorname{lncRNA-miRNA-Gene~Interaction~}$}

We next studied the interaction between lncRNA and miRNA using the prediction tool miRanda. For this purpose, the miRNA sequences were downloaded from the mirbase database. A total of 6011 interactions between 10 lncRNA and 2175 miRNA were observed. From the miRCancer database, cancer-related miRNAs were downloaded, and $654 \mathrm{BC}$ related miRNA candidates were identified. There were 118 miRNAs common between 654 BC-related miRNAs and 2175 miRNAs that were observed in the interaction network. The interactions for these 118 miRNAs were extracted from the total 6011 interactions, and a total of 315 interactions were observed. Then, a lncRNA-miRNA-gene network was constructed using Cytoscape. The genes in the network were the host genes of the lncRNAs. From this network, a subnetwork was constructed with the top five hub lncRNAs (Figure 4). The deepness of the red color indicates a higher degree of lncRNA. Here, the degree of a lncRNA means the number of miRNAs and genes connected to that lncRNA. From the network, it was observed that two genes, BID and KLF10 (pink colored), were functionally related to BC. Two lncRNAs, TCONS_00076394 and TCONS_00051377, were interacted with BC-related miRNAs and their host genes were also functionally related to BC. This indicated that these two lncRNAs might be important biomarkers for TNBC.

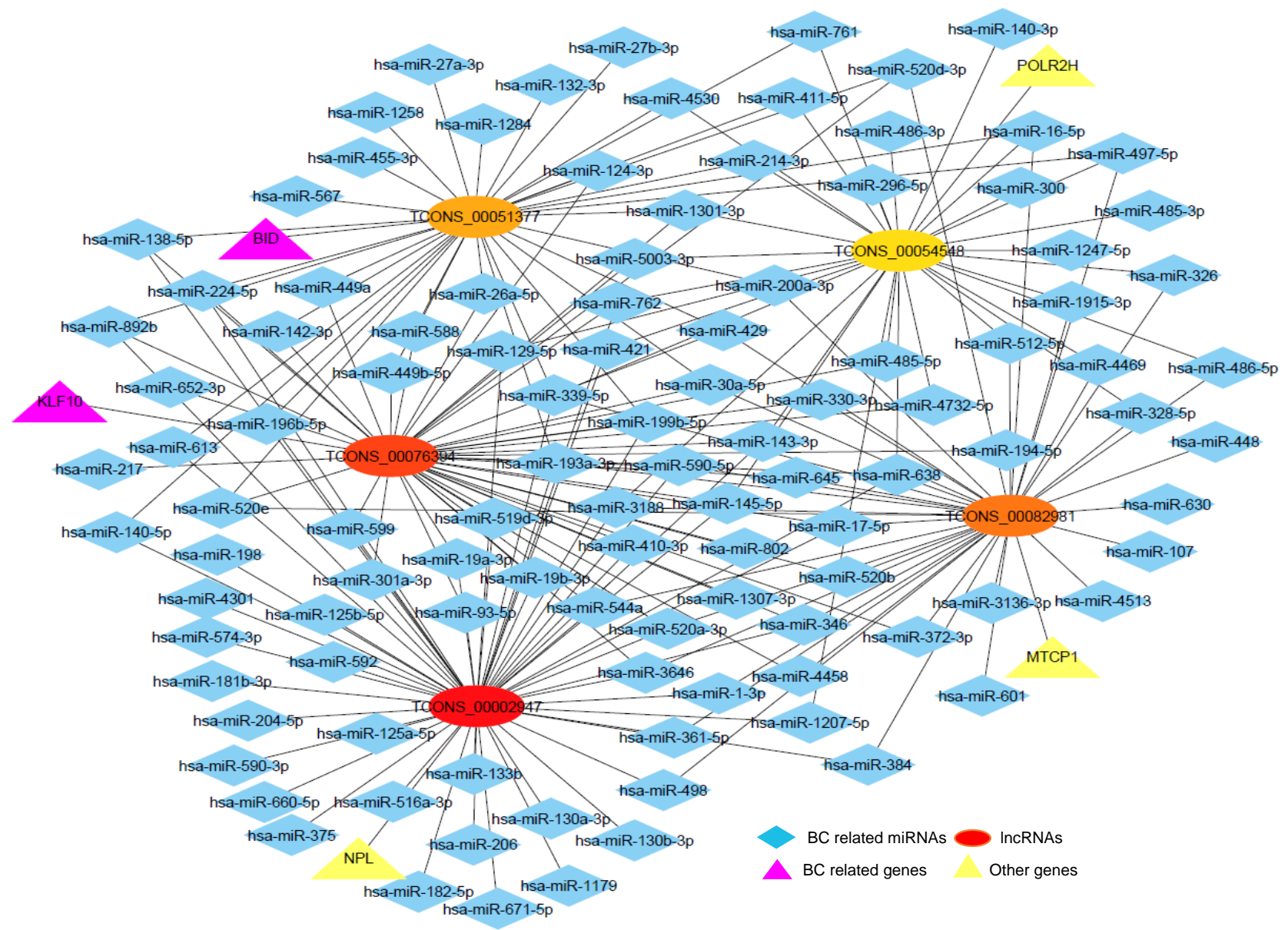

Figure 4. LncRNA-miRNA-gene interaction network. The lncRNAs are the top 5 hub lncRNAs where the deepness of the red color indicates a higher degree of lncRNAs. All the miRNAs are related with BC. The genes are the host genes of the lncRNAs.

\subsection{GO Term and KEGG Pathway Enrichment Analysis}

We downloaded the PPI network from the STRING database for the 197 host genes of the DE lncRNA transcripts and found that 35 genes participated in the PPI network. 
We then performed the GO and KEGG pathways analyses for these genes. The GO term biological process analysis showed that some of the host genes were involved with the positive regulation of cell division, neutrophil chemotaxis, creatine metabolism, positive regulation of protein phosphorylation, defense response to protozoan, and negative regulation of cell proliferation. The GO term molecular function analysis showed that host genes were enriched in transferase activity, transferring phosphorus-containing groups, kinase activity, creatine kinase activity, and growth factor activity. The GO term cellular component analysis showed that the host genes were enriched in the mitochondrial inner membrane. The KEGG pathway analysis showed that some of the genes were involved in the prostate cancer pathway. The significant GO term and pathways were shown in Table 4.

Table 4. Significant GO terms and pathways of 35 genes from the PPI networks of host genes of the DE lncRNAs.

\begin{tabular}{|c|c|c|c|}
\hline GO/Pathway ID & GO/Pathway Name & No. of Genes & $p$-Value \\
\hline \multicolumn{4}{|c|}{ Biological Process } \\
\hline GO:0051781 & Positive regulation of cell division & 3 & 0.0036 \\
\hline GO:0030593 & Neutrophil chemotaxis & 3 & 0.0070 \\
\hline GO:0006600 & Creatine metabolic process & 2 & 0.0208 \\
\hline GO:0001934 & $\begin{array}{l}\text { Positive regulation of protein } \\
\text { phosphorylation }\end{array}$ & 3 & 0.0243 \\
\hline GO:0042832 & Defense response to protozoan & 2 & 0.0356 \\
\hline GO:0008285 & $\begin{array}{l}\text { Negative regulation of cell proliferation } \\
\text { Cellular Component }\end{array}$ & 4 & 0.0390 \\
\hline \multicolumn{3}{|c|}{ Molecular Function } & 0.0449 \\
\hline GO:0016772 & $\begin{array}{l}\text { Transferase activity, transferring } \\
\text { phosphorus-containing groups }\end{array}$ & 2 & 0.0094 \\
\hline GO:0016301 & Kinase activity & 4 & 0.0105 \\
\hline GO:0004111 & Creatine kinase activity & 2 & 0.0113 \\
\hline \multicolumn{4}{|c|}{ KEGG pathway } \\
\hline hsa05215 & Prostate cancer & 3 & 0.0265 \\
\hline
\end{tabular}

\section{Discussion}

TNBC is well-known as a major problem affecting the health of females worldwide and its underlying mechanism has not been revealed properly. Due to the outstanding heterogeneity of $\mathrm{BCs}$, it is crucial not only to categorize them based on morphologic and clinical features but also to examine intrinsic molecular signatures. Compared to other BCs, TNBC is regarded as high malignancy, young-onset, easy recurrence, and low survival rates [42,43]. Since TNBC is deficient in ER, PR and HER2 receptors, this type of cancer has no specific targets of hormone therapy as well as a targeted treatment. In this regard, it is essential to detect alternative molecular targets for TNBC treatment. To this line, we aimed to investigate potential lncRNAs interrelated with TNBC.

In this study, single-cell RNA-Seq data were used to identify the changes in genomic expression profiles of lncRNA as well as mRNA in patients with TNBC. A comprehensive bioinformatics pipeline was adopted from the previous literature [44-46] for the detection of novel lncRNAs in TNBC patients. The potential biological functions of the newly identified lncRNA candidates were inferred by identifying the functional importance of adjacent protein-coding genes along with their co-expression profiles. This study provides novel statistics about the early immunity against TNBC and figures out the significance of regulatory RNAs.

Here, a total of 83,401 transcripts of which 10,385 transcripts with a class code " $\mathrm{j}$ " having unknown annotation were applied for the detection of the putative lncRNAs. After the filtration process, a total of 909 candidate lncRNAs were identified. Out of 909 candidate 
lncRNA transcripts, 19 were significantly DE of which three were upregulated and 16 were downregulated.

Previous studies have explored the particular role of lncRNAs in TNBC. MALAT1, for instance, has been reported as a promoter of proliferation and invasion through microRNA129-5p [32]. The overexpression of IncRNA MIR100HG was related to poor prognosis while downregulation of MIR100HG significantly suppressed TNBC-induced cell proliferation, and reduced tumor growth [35]. Our identified lncRNAs candidates showed differential expression in normal vs. tumor groups. In addition, owing to the inherent regulatory nature, lncRNAs may have a greater biological impact compared to a single gene. Even the candidate lncRNAs might be associated with the TNBC by sponging miRNAs. As we identified the candidate lncRNAs through the computational approach, knowing more specific roles of the candidate lncRNAs through the experimental procedure is beyond the scope of this study. Further experiments are needed to explore more specific roles of the identified candidates.

LncRNAs have been shown to affect the expression, or function of their host gene [47]. We predicted the function of IncRNAs by GO analysis for the host genes of the differentially expressed lncRNAs. GO term analyses showed that several host genes functioned in some important biological processes, molecular mechanisms and cellular components. The KEGG pathway analysis showed that the host genes were involved in the prostate cancer pathway (Table 4).

The most important property of lncRNA is known to have a function as miRNA sponges [48]. As mRNAs play a crucial role in cancer progression, we performed lncRNAmiRNA-gene interaction network analysis to explore the relationship between the DE lncRNAs and miRNAs. From the network analysis, it was found that the five hub lncRNAs interacted with the BC-related miRNAs.

The host gene of the lncRNAs was also related functionally to BC. Two host genes BID and KLF10 were related to BC. BID was found to be a potential target in the therapeutic strategies for BC [49]. KLF10 was an anti-metastasis gene that significantly prevents BC cell invasion [50]. KLF10 was involved in cell proliferation and apoptosis, and its levels were inversely associated with $\mathrm{BC}$ stages, implying that it had a tumor suppressor function [51]. The host genes BID and KLF10 were the important biomarkers for BC. Similarly, the lncRNAs TCONS_00076394 and TCONS_00051377 were interacted with BCrelated miRNAs. These two lncRNAs were unannotated which meant that their function was unknown. As the function of lncRNAs can be predicted based on the function of their interacted miRNAs and host genes, TCONS_00076394 and TCONS_00051377, might be the potential biomarkers for BC. However, there are some limitations in this study. Firstly, the datasets that had been availed here to detect the DE candidate lncRNAs and mRNAs were adopted from a public resource as well as the fact that they were not experimentally validated. Secondly, owing to the lower sample size, pairwise comparisons of some parameters were not statistically significant enough. So that further experiments might be effective to validate our findings.

\section{Materials and Methods}

\subsection{Data Collection}

The scRNA-seq FastQ data of six patients with TNBC (PT039, PT058, PT081, PT084, PT089, and PT126) were downloaded from the public resources Sequence Read Archive (SRA) database (GEO: GSE118390) [52]. In addition, 20 normal breast epithelial cells were also downloaded, which acted as reference cells to compare differential lncRNAs expression between normal and diseases states [53]. In this study, a total of 60 SRR files of six TNBC patients (10 from each) were included.

\subsection{Quality Control, Mapping, and Transcript Assembly}

The first step of putative RNA analysis involves sequence alignment and transcript assembly. FastQC was applied to check data quality and only good quality data were 
included for analyses. Sequence alignment was performed against the reference human genome (hg38) using STAR (Version STAR-2.7.9a) to search match reads and to merge duplicated and low-quality reads [54]. These data were subsequently served as input for Cufflinks for transcript assembly [55]. Gene transfer files (gtf) generated by Cufflinks were merged by Cuffmerge. Cufflinks gffread was applied to fetch fasta files from merged gtf files. To achieve the overall assembly quality, the human genome along with the annotation files were provided as inputs to Cuffmerge.

\subsection{Transcripts Filtration}

Fasta files containing less than 200 nucleotides were discarded to find out potential lncRNAs from the transcripts produced by Cufflinks. It has been demonstrated that several lncRNAs contain only single-exon transcripts $[44,56,57]$. These single-exon transcripts; however, are often removed since they can lead to generating background noise as a result of inaccurate transcript assembly, experimental artifacts, or genomic contamination during sequence library preparation. To overcome this problem, Sun et al. [58] employed a stringent size threshold of $1000 \mathrm{bp}$ for identifying single-exon transcripts. Later, Bush et al. [59] used a size threshold of $500 \mathrm{bp}$ that we applied in this assay, considering the shortcomings and the point that the mean length of single-exon transcript in humans is reported to be $<300 \mathrm{bp}$ [60]. The full-length sequences for all transcripts (obtained from Cufflinks gffread) were considered as the reference genome and the fastq reads were mapped using the bowtie2 aligner. Then, count data was generated using bedtools multiBamCov with the output of bowtie2 (converted to bam, sorted, and indexed). The count data was used to identify differentially expressed (DE) transcripts by the R package DESeq. Foldchange $>2$ and $p$-value $<0.05$ were considered as the cut-off for defining significant DE transcripts.

\subsection{Assessment of the Coding Potential}

In this study, protein-coding potential (PCP) of shortlisted lncRNAs were checked using a coding-potential-calculator 2 (CPC2) [61]. CPC2 uses sequence hallmark to differentiate between coding and non-coding RNAs, hence, DNA sequences of the transcripts were extracted applying Cufflink's gffread [59]. A cut-off coding probability of $p<0.5$ for CPC2 was chosen to treat a transcript as non-coding and to decrease false-positive identification [44]. The detailed pipeline of identifying lncRNA candidates is depicted in Figure 5.

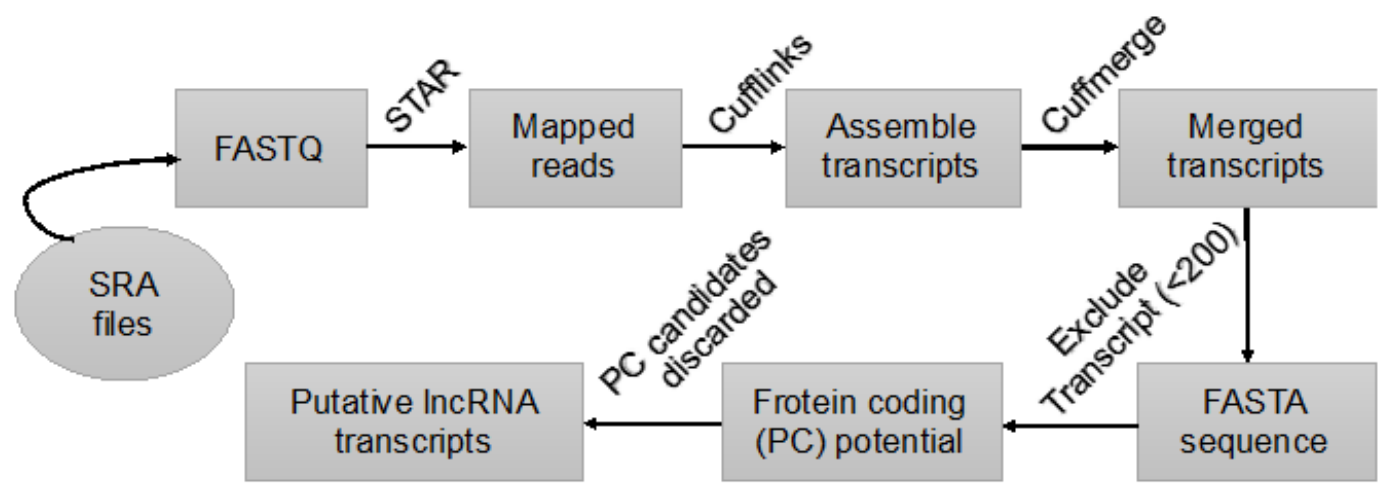

Figure 5. In silico identification method of lncRNAs from scRNA-seq databases.

\subsection{Differential Expression Analysis}

Differential expression analysis was done using DESeq [62]. The count data (obtained from multiBamCov-bedtools 2.30.0) was used to identify differentially expressed (DE) transcripts between the normal and TNBC group. Foldchange $(>2)$ and $p$-value $(<0.05)$ were considered as the cut-off for defining significant DE transcripts. A heatmap was used to differentiate the expression patterns between normal and cancer groups. 


\subsection{GO and KEGG Enrichment Analysis}

After finding the DE lncRNAs, the host genes of the DE lncRNA transcripts were extracted. The host genes of DE lncRNAs were mapped to Search Tool for the Retrieval of Interacting Genes (STRING v11.5; http://string-db.org/cgi/input.pl, accessed on 3 August 2021), and the protein-protein interaction (PPI) among the genes was obtained. Then, a PPI network was constructed using the software Cytoscape. From the network, the top hub genes were selected for GO term and KEGG pathway enrichment analyses. With the selected hub genes, Gene Ontology (GO) term and KEGG pathway enrichment analyses were performed using DAVID to know the function of the lncRNAs. The threshold $p$-value $<0.05$ was used for the significance of the enrichment analysis.

\subsection{LncRNA-miRNA-Gene Interaction Network Analysis}

The DE lncRNAs were sorted based on $p$-value and fold change. Then, the top $10 \mathrm{DE}$ lncRNAs (three upregulated and seven downregulated) were selected for miRNA interaction analysis. The lncRNA-miRNA interaction was predicted using miRanda software. The miRNA sequences were downloaded from mirbase. From the miRCancer database, the miRNAs related to $\mathrm{BC}$ were also downloaded. From the lncRNA-miRNA interaction network, a sub-network was constructed keeping only the BC-related miRNAs. Finally, the lncRNA-miRNA-gene network was constructed using Cytoscape where the genes were the host genes of the DE lncRNAs.

\section{Conclusions}

In this study, we investigated potential lncRNAs interrelated with TNBC using singlecell RNA-Seq data and found two lncRNAs TCONS_00076394 and TCONS_00051377 as potential biomarkers for TNBC. These two lncRNAs were differentially expressed between cancer and normal groups, interacted with breast cancer-related miRNAs, and their host genes were also functionally related to breast cancer. Thus, we can conclude that these two lncRNAs TCONS_00076394 and TCONS_00051377 might be considered as potential biomarkers for TNBC. Further experiments are required to know the specific function of these lncRNAs in TNBC.

Author Contributions: T.H. and M.R. designed the experiment, performed the bioinformatics pipelines, analyzed the data, wrote the manuscript and drew the figures and tables. S.R., Y.P., S.F. and Y.W. extensively edited the manuscript. S.F. and Y.W. supervised the work. All the authors read and approved the final version of the manuscript for publication. All authors have read and agreed to the published version of the manuscript.

Funding: This work was partly supported by the National Key Research and Development Program of China under Grant No. 2018YFB0204403; Strategic Priority CAS Project XDB38050100; National Science Foundation of China under grant no. U1813203; the Shenzhen Basic Research Fund under grant no. JCYJ20200109114818703, RCYX2020071411473419, and JSGG20201102163800001; CAS Key Lab under grant no. 2011DP173015 (YW). We would also like to thank the funding support from the Youth Innovation Promotion Association, CAS to Yanjie Wei.

Acknowledgments: We would like thank all the members of Computational Biology and Bioinformatics Lab, Center for High Performance Computing, SIAT, CAS for their valuable suggestions and feedbacks.

Conflicts of Interest: The authors declare no conflict of interest. The funders had no role in the design of the study; in the collection, analyses, or interpretation of data; in the writing of the manuscript, or in the decision to publish the results.

\section{References}

1. Chen, W.; Zheng, R.; Baade, P. Cancer statistics in China. CA Cancer J. Clin. 2016, 66, 115-132. [CrossRef] [PubMed]

2. Bray, F.; McCarron, P.; Parkin, D.M. The changing global patterns of female breast cancer incidence and mortality. Breast Cancer Res. 2004, 6, 229-239. [CrossRef] [PubMed] 
3. Liu, L.; Zhang, Y.; Lu, J. The roles of long noncoding RNAs in breast cancer metastasis. Cell Death Dis. 2020, 11, 749. [CrossRef] [PubMed]

4. Gupta, G.P.; Massagué, J. Cancer metastasis: Building a framework. Cell 2006, 127, 679-695. [CrossRef] [PubMed]

5. Hanahan, D.; Weinberg, R.A. Hallmarks of cancer: The next generation. Cell 2011, 144, 646-674. [CrossRef]

6. Koboldt, D.C.; Fulton, R.S.; McLellan, M.D.; Schmidt, H.; Kalicki-Veizer, J.; McMichael, J.F.; Fulton, L.L.; Dooling, D.J.; Ding, L.; Mardis, E.R.; et al. Comprehensive molecular portraits of human breast tumours. Nature 2012, 490, 61-70. [CrossRef]

7. Perou, C.M.; Sørile, T.; Eisen, M.B.; Van De Rijn, M.; Jeffrey, S.S.; Ress, C.A.; Pollack, J.R.; Ross, D.T.; Johnsen, H.; Akslen, L.A.; et al. Molecular portraits of human breast tumours. Nature 2000, 406, 747-752. [CrossRef]

8. Rakha, E.A.; El-Sayed, M.E.; Green, A.R.; Lee, A.H.S.; Robertson, J.F.; Ellis, I.O. Prognostic markers in triple-negative breast cancer. Cancer 2007, 109, 25-32. [CrossRef]

9. Pusztai, L.; Karn, T.; Safonov, A.; Abu-Khalaf, M.M.; Bianchini, G. New strategies in breast cancer: Immunotherapy. Clin. Cancer Res. 2016, 22, 2105-2110. [CrossRef]

10. Klinge, C.M. Non-coding RNAs in breast cancer: Intracellular and intercellular communication. Non-Coding RNA 2018, 4, 40. [CrossRef] [PubMed]

11. Zhou, S.; He, Y.; Yang, S.; Hu, J.; Zhang, Q.; Chen, W.; Xu, H.; Zhang, H.; Zhong, S.; Zhao, J.; et al. The regulatory roles of lncRNAs in the process of breast cancer invasion and metastasis. Biosci. Rep. 2018, 38, BSR20180772. [CrossRef] [PubMed]

12. Tomar, D.; Yadav, A.S.; Kumar, D.; Bhadauriya, G.; Kundu, G.C. Non-coding RNAs as potential therapeutic targets in breast cancer. Biochim. Biophys. Acta Gene Regul. Mech. 2020, 1863, 194378. [CrossRef]

13. Bin, X.; Hongjian, Y.; Xiping, Z.; Bo, C.; Shifeng, Y.; Binbin, T. Research progresses in roles of LncRNA and its relationships with breast cancer. Cancer Cell Int. 2018, 18, 179. [CrossRef]

14. Bhan, A.; Soleimani, M.; Mandal, S.S. Long noncoding RNA and cancer: A new paradigm. Cancer Res. 2017, 77, 3965-3981. [CrossRef] [PubMed]

15. Iyer, M.K.; Niknafs, Y.S.; Malik, R.; Singhal, U.; Sahu, A.; Hosono, Y.; Barrette, T.R.; Prensner, J.R.; Evans, J.R.; Zhao, S.; et al. The landscape of long noncoding RNAs in the human transcriptome. Nat. Genet. 2015, 47, 199-208. [CrossRef]

16. Zhang, T.; Hu, H.; Yan, G.; Wu, T.; Liu, S.; Chen, W.; Ning, Y.; Lu, Z. Long non-coding RNA and breast cancer. Technol. Cancer Res. Treat. 2019, 18, 1-10. [CrossRef]

17. Peng, W.X.; Koirala, P.; Mo, Y.Y. LncRNA-mediated regulation of cell signaling in cancer. Oncogene 2017, 36, 5661-5667. [CrossRef]

18. Li, Z.; Hou, P.; Fan, D.; Dong, M.; Ma, M.; Li, H.; Yao, R.; Li, Y.; Wang, G.; Geng, P.; et al. The degradation of EZH2 mediated by lncRNA ANCR attenuated the invasion and metastasis of breast cancer. Cell Death Differ. 2017, 24, 59-71. [CrossRef] [PubMed]

19. Lou, K.X.; Li, Z.H.; Wang, P.; Liu, Z.; Chen, Y.; Wang, X.L.; Cui, H.X. Long non-coding RNA BANCR indicates poor prognosis for breast cancer and promotes cell proliferation and invasion. Eur. Rev. Med. Pharmacol. Sci. 2018, 22, 1358-1365. [CrossRef]

20. Li, W.; Jia, G.; Qu, Y.; Du, Q.; Liu, B.; Liu, B. Long non-coding RNA (LncRNA) HOXA11-AS promotes breast cancer invasion and metastasis by regulating epithelial-mesenchymal transition. Med. Sci. Monit. 2017, 23, 3393-3403. [CrossRef] [PubMed]

21. Hansji, H.; Leung, E.Y.; Baguley, B.C.; Finlay, G.J.; Askarian-Amiri, M.E. Keeping abreast with long non-coding RNAs in mammary gland development and breast cancer. Front. Genet. 2014, 5, 379. [CrossRef]

22. Tripathi, M.K.; Doxtater, K.; Keramatnia, F.; Zacheaus, C.; Yallapu, M.M.; Jaggi, M.; Chauhan, S.C. Role of lncRNAs in ovarian cancer: Defining new biomarkers for therapeutic purposes. Drug Discov. Today 2018, 23, 1635-1643. [CrossRef] [PubMed]

23. Wong, C.M.; Tsang, F.H.; Ng, I.O.L. Non-coding RNAs in hepatocellular carcinoma: Molecular functions and pathological implications. Nat. Rev. Gastroenterol. Hepatol. 2018, 15, 137-151. [CrossRef]

24. Alvarez-Dominguez, J.R.; Lodish, H.F. Emerging mechanisms of long noncoding RNA function during normal and malignant hematopoiesis. Blood 2017, 130, 1965-1975. [CrossRef] [PubMed]

25. Collina, F.; Aquino, G.; Brogna, M.; Cipolletta, S.; Buonfanti, G.; De Laurentiis, M.; Di Bonito, M.; Cantile, M.; Botti, G. LncRNA HOTAIR up-regulation is strongly related with lymph nodes metastasis and LAR subtype of triple negative breast cancer. J. Cancer 2019, 10, 2018-2024. [CrossRef]

26. Haffty, B.G.; Yang, Q.; Reiss, M.; Kearney, T.; Higgins, S.A.; Weidhaas, J.; Harris, L.; Hait, W.; Toppmeyer, D. Locoregional relapse and distant metastasis in conservatively managed triple negative early-stage breast cancer. J. Clin. Oncol. 2006, $24,5664$. [CrossRef]

27. Chen, V.E.; Gillespie, E.F.; Zakeri, K.; Murphy, J.D.; Yashar, C.M.; Lu, S.; Einck, J.P. Pathologic response after neoadjuvant chemotherapy predicts locoregional control in patients with triple negative breast cancer. Adv. Radiat. Oncol. 2017, 2, 105-109. [CrossRef]

28. Fan, H.; Yuan, J.; Li, X.; Ma, Y.; Wang, X.; Xu, B.; Li, X. LncRNA LINC00173 enhances triple-negative breast cancer progression by suppressing miR-490-3p expression. Biomed. Pharmacother. 2020, 125, 109987. [CrossRef] [PubMed]

29. Han, C.; Fu, Y.; Zeng, N.; Yin, J.; Li, Q. LncRNA FAM83H-AS1 promotes triple-negative breast cancer progression by regulating the miR-136-5p/metadherin axis. Aging 2020, 12, 3594. [CrossRef]

30. Hua, K.; Deng, X.; Hu, J.; Ji, C.; Yu, Y.; Li, J.; Wang, X.; Fang, L. Long noncoding RNA HOST2, working as a competitive endogenous RNA, promotes STAT3-mediated cell proliferation and migration via decoying of let-7b in triple-negative breast cancer. J. Exp. Clin. Cancer Res. 2020, 39, 1-13. [CrossRef] [PubMed]

31. Yang, F.; Dong, S.Y.; Lv, L.; Liu, Y.H.; Yao, Z.H.; Zhang, X.H.; Wang, O.C. Long non-coding RNA AFAP1-AS1 was up-regulated in triple-negative breast cancer and regulated proliferation and invasion. Int. J. Clin. Exp. Pathol. 2016, 9, 6378-6384. 
32. Zuo, Y.; Li, Y.; Zhou, Z.; Ma, M.; Fu, K. Long non-coding RNA MALAT1 promotes proliferation and invasion via targeting miR-129-5p in triple-negative breast cancer. Biomed. Pharmacother. 2017, 95, 922-928. [CrossRef] [PubMed]

33. Niu, L.; Fan, Q.; Yan, M.; Wang, L. LncRNA NRON down-regulates lncRNA snaR and inhibits cancer cell proliferation in TNBC. Biosci. Rep. 2019, 39, BSR20190468. [CrossRef]

34. Wang, L.; Liu, D.; Wu, X.; Zeng, Y.; Li, L.; Hou, Y.; Li, W.; Liu, Z. Long non-coding RNA (LncRNA) RMST in triple-negative breast cancer (TNBC): Expression analysis and biological roles research. J. Cell. Physiol. 2018, 233, 6603-6612. [CrossRef] [PubMed]

35. Wang, S.; Ke, H.; Zhang, H.; Ma, Y.; Ao, L.; Zou, L.; Yang, Q.; Zhu, H.; Nie, J.; Wu, C.; et al. LncRNA MIR100HG promotes cell proliferation in triple-negative breast cancer through triplex formation with p27 loci. Cell Death Dis. 2018, 9, 1-11. [CrossRef] [PubMed]

36. Luo, L.; Tang, H.; Ling, L.; Li, N.; Jia, X.; Zhang, Z.; Wang, X.; Shi, L.; Yin, J.; Qiu, N.; et al. LINC01638 lncRNA activates MTDH-Twist1 signaling by preventing SPOP-mediated c-Myc degradation in triple-negative breast cancer. Oncogene 2018, 37, 1178. [CrossRef]

37. Liang, Y.; Hu, J.; Li, J.; Liu, Y.; Yu, J.; Zhuang, X.; Mu, L.; Kong, X.; Hong, D.; Yang, Q.; et al. Epigenetic activation of TWIST1 by MTDH promotes cancer stem-like cell traits in breast cancer. Cancer Res. 2015, 75, 3672-3680. [CrossRef]

38. Wang, O.C.; Yang, F.; Liu, Y.H.; Lv, L.; Ma, R.; Chen, C.; Wang, J.; Tan, Q.; Cheng, Y.; Xia, E.; et al. C-MYC-induced upregulation of lncRNA SNHG12 regulates cell proliferation, apoptosis and migration in triple-negative breast cancer. Am. J. Transl. Res. 2017, 9, 533.

39. Cech, T.R.; Steitz, J.A. The noncoding RNA revolution-Trashing old rules to forge new ones. Cell 2014, 157, 77-94. [CrossRef]

40. Pang, K.C.; Frith, M.C.; Mattick, J.S. Rapid evolution of noncoding RNAs: Lack of conservation does not mean lack of function. Trends Genet. 2006, 22, 1-5. [CrossRef]

41. Weikard, R.; Demasius, W.; Kuehn, C. Mining long noncoding RNA in livestock. Anim. Genet. 2017, 48, 3-18. [CrossRef] [PubMed]

42. Dent, R.; Trudeau, M.; Pritchard, K.I.; Hanna, W.M.; Kahn, H.K.; Sawka, C.A.; Lickley, L.A.; Rawlinson, E.; Sun, P.; Narod, S.A. Triple-negative breast cancer: Clinical features and patterns of recurrence. Clin. Cancer Res. 2007, 13, 4429-4434. [CrossRef] [PubMed]

43. Li, X.X.; Wang, L.J.; Hou, J.; Liu, H.Y.; Wang, R.; Wang, C.; Xie, W.H. Identification of long noncoding RNAs as predictors of survival in triple-negative breast cancer based on network analysis. Biomed. Res. Int. 2020, 2020, 8970340. [CrossRef] [PubMed]

44. Weikard, R.; Hadlich, F.; Kuehn, C. Identification of novel transcripts and noncoding RNAs in bovine skin by deep next generation sequencing. BMC Genom. 2013, 14, 789. [CrossRef]

45. Billerey, C.; Boussaha, M.; Esquerré, D.; Rebours, E.; Djari, A.; Meersseman, C.; Klopp, C.; Gautheret, D.; Rocha, D. Identification of large intergenic non-coding RNAs in bovine muscle using next-generation transcriptomic sequencing. BMC Genom. 2014, 15, 499. [CrossRef]

46. Gupta, P.; Peter, S.; Jung, M.; Lewin, A.; Hemmrich-Stanisak, G.; Franke, A.; von Kleist, M.; Schütte, C.; Einspanier, R.; Sharbati, S.; et al. Analysis of long non-coding RNA and mRNA expression in bovine macrophages brings up novel aspects of Mycobacterium avium subspecies paratuberculosis infections. Sci. Rep. 2019, 9, 1571. [CrossRef]

47. Delás, M.J.; Hannon, G.J. lncRNAs in development and disease: From functions to mechanisms. Open Biol. $2017,7,170121$. [CrossRef]

48. Li, Z.; Zhao, W.; Wang, M.; Zhou, X. The Role of Long Noncoding RNAs in Gene Expression Regulation; IntechOpen: London, UK, 2019.

49. Bonofiglio, D.; Cione, E.; Vizza, D.; Perri, M.; Pingitore, A.; Qi, H.; Catalano, S.; Rovito, D.; Genchi, G.; Andò, S. Bid as a potential target of apoptotic effects exerted by low doses of PPAR $\gamma$ and RXR ligands in breast cancer cells. Cell Cycle 2011, 10, 2344-2354. [CrossRef] [PubMed]

50. Memon, A.; Lee, W.K. KLF10 as a tumor suppressor gene and its TGF- $\beta$ signaling. Cancers 2018, 10, 161. [CrossRef] [PubMed]

51. Subramaniam, M.; Hawse, J.R.; Rajamannan, N.M.; Ingle, J.N.; Spelsberg, T.C. Functional role of KLF10 in multiple disease processes. BioFactors 2010, 36, 8-18. [CrossRef] [PubMed]

52. Karaayvaz, M.; Cristea, S.; Gillespie, S.M.; Patel, A.P.; Mylvaganam, R.; Luo, C.C.; Specht, M.C.; Bernstein, B.E.; Michor, F.; Ellisen, L.W. Unravelling subclonal heterogeneity and aggressive disease states in TNBC through single-cell RNA-seq. Nat. Commun. 2018, 9, 3588. [CrossRef]

53. Gao, R.; Kim, C.; Sei, E.; Foukakis, T.; Crosetto, N.; Chan, L.K.; Srinivasan, M.; Zhang, H.; Meric-Bernstam, F.; Navin, N. Nanogrid single-nucleus RNA sequencing reveals phenotypic diversity in breast cancer. Nat. Commun. 2017, 8, 228. [CrossRef]

54. Dobin, A.; Davis, C.A.; Schlesinger, F.; Drenkow, J.; Zaleski, C.; Jha, S.; Batut, P.; Chaisson, M.; Gingeras, T.R. STAR: Ultrafast universal RNA-seq aligner. Bioinformatics 2013, 29, 15-21. [CrossRef] [PubMed]

55. Trapnell, C.; Roberts, A.; Goff, L.; Pertea, G.; Kim, D.; Kelley, D.R.; Pimentel, H.; Salzberg, S.L.; Rinn, J.L.; Pachter, L. Differential gene and transcript expression analysis of RNA-seq experiments with TopHat and Cufflinks. Nat. Protoc. 2012, 7, 562-578. [CrossRef]

56. Koufariotis, L.T.; Chen, Y.P.P.; Chamberlain, A.; Jagt, C.V.; Hayes, B.J. A catalogue of novel bovine long noncoding RNA across 18 tissues. PLoS ONE 2015, 10, e0141225. [CrossRef]

57. Jorquera, R.; Ortiz, R.; Ossandon, F.; Cárdenas, J.P.; Sepúlveda, R.; González, C.; Holmes, D.S. SinEx DB: A database for single exon coding sequences in mammalian genomes. Database 2016, 2016, 1-8. [CrossRef]

58. Sun, M.; Gadad, S.S.; Kim, D.S.; Kraus, W.L. Discovery, annotation, and functional analysis of long noncoding RNAs controlling cell-cycle gene expression and proliferation in breast cancer cells. Mol. Cell 2015, 59, 698-711. [CrossRef] 
59. Bush, S.J.; Muriuki, C.; McCulloch, M.E.B.; Farquhar, I.L.; Clark, E.L.; Hume, D.A. Cross-species inference of long non-coding RNAs greatly expands the ruminant transcriptome. Genet. Sel. Evol. 2018, 50, 20. [CrossRef] [PubMed]

60. Rédei, G.P. Encyclopedia of genetics, genomics, proteomics and informatics. In Encyclopedia of Genetics, Genomics, Proteomics and Informatics; Springer: Berlin/Heidelberg, Germany, 2008; p. 285.

61. Kong, L.; Zhang, Y.; Ye, Z.Q.; Liu, X.Q.; Zhao, S.Q.; Wei, L.; Gao, G. CPC: Assess the protein-coding potential of transcripts using sequence features and support vector machine. Nucleic Acids Res. 2007, 35, 345-349. [CrossRef] [PubMed]

62. Love, M.I.; Huber, W.; Anders, S. Moderated estimation of fold change and dispersion for RNA-seq data with DESeq2. Genome Biol. 2014, 15, 550. [CrossRef] 